\title{
On the efficiency of drawing computer-generated line stimuli: Rectangles can be faster than lines
}

\author{
CHRISTIAN N. L. OLIVERS \\ University of Birmingham, Birmingham, England \\ and \\ DERRICK G. WATSON \\ University of Warwick, Coventry, England
}

\begin{abstract}
A simple method is presented for optimizing the efficiency of drawing line stimuli in Turbo Pascal. It involves the substitution of Rectangle and Bar commands for Line commands and results in time savings of up to $90 \%$. The savings are most consistent for horizontal lines, although some savings may also be made for vertical lines. The effects of different graphics libraries, graphics modes, and hardware configurations are discussed. The method proves especially useful in improving the spatiotemporal resolution of on-line-generated motion displays.
\end{abstract}

In 1954, Bitterman, Krauskopf, and Hochberg reported a new technique of creating visual shapes. They cut out various pieces of paper, impregnated them with an inhibitor of bacterial growth, and lay them in a Petri dish filled with a bacterial culture. The inhibitor diffused from the paper into the culture, creating visible differences in bacterial densities. Nowadays, thankfully, vision researchers can resort to a much more versatile and powerful tool for presenting visual stimuli: the computer, in combination with the scanning cathode ray tube. Modern computers can be flexibly applied to present all kinds of stimuli, ranging from primitive visual shapes, such as lines and circles, to high resolution video clips. However, computer hardware also imposes certain limits on the flexibility with which visual stimuli can be created.

In outline, the process of presenting a picture on the computer screen follows two basic steps: (1) The computer program issues image-drawing commands that result in stimuli being drawn in the video memory (RAM) of the computer's video card, and (2) the monitor's electron beam draws the image stored in video memory on the screen line by line from top to bottom. Several hardware limitations constrain the type of stimuli that can be presented. Video screens (and cards) have limited spatial and temporal, as well as color and luminance, resolutions (see Bach, Meigen, \& Strasburger, 1997, for an overview). For example, the limited pixel size causes oblique lines to appear jagged (but see Tanner, Jolicoeur, Cowan, Booth, \& Fish-

We thank Chris Howard from Genus Microprogramming for valuable comments and Andrew Olson for providing a $\mathrm{C}++$ example. Correspondence concerning this article should be addressed to C. N. L. Olivers, Cognitive Science Research Centre, School of Psychology, University of Birmingham, Edgbaston, Birmingham, B15 2TT England (e-mail: c.n.olivers@bham.ac.uk). man, 1989, for a solution). An important temporal limitation is the scanning rate of the computer's video card and monitor. The process of displaying an image on the screen takes a considerable amount of time, as the electron beam moves serially from the upper left-hand corner to the lower right-hand corner, scanning/tracing one horizontal line after the other. When the beam has reached the bottom, it flies back to the top and starts again (this is called the vertical retrace). This entire process takes between 11 and $17 \mathrm{msec}$, depending on the scan rate (usually around $70 \mathrm{~Hz}$ ). In the present paper, we will discuss the implications of the scan period for the presentation of moving displays and will demonstrate a method that can improve drawing speed.

\section{Synchronizing Drawing With the Screen Retrace}

Ideally, stimulus drawing should be synchronized with the screen retrace (see, e.g., Haussmann, 1992). For instance, the drawing algorithm should wait until the monitor's electron beam is in retrace or is at the top of the screen and then fully draw the stimulus in video memory before the monitor's beam starts to actually display it on the screen. Otherwise, the onset and offset of (part of) an image can be delayed by up to a full scan period. If one is interested in the time required for a subject to respond to the stimulus, this delay in presentation would lead to timing inaccuracies. In addition, the bottom part of the stimulus could be displayed on the monitor before the top half. This is especially harmful to the quality of motion stimuli, as is illustrated in an example from Watson and Humphreys (1997; see Figure 1). They presented subjects with colored letter Hs that moved continuously downward, bounded by a virtual window. Note that computer-generated motion stimuli do not contain real movement. They are just a series of snapshots slightly different in spatial layout, pro- 

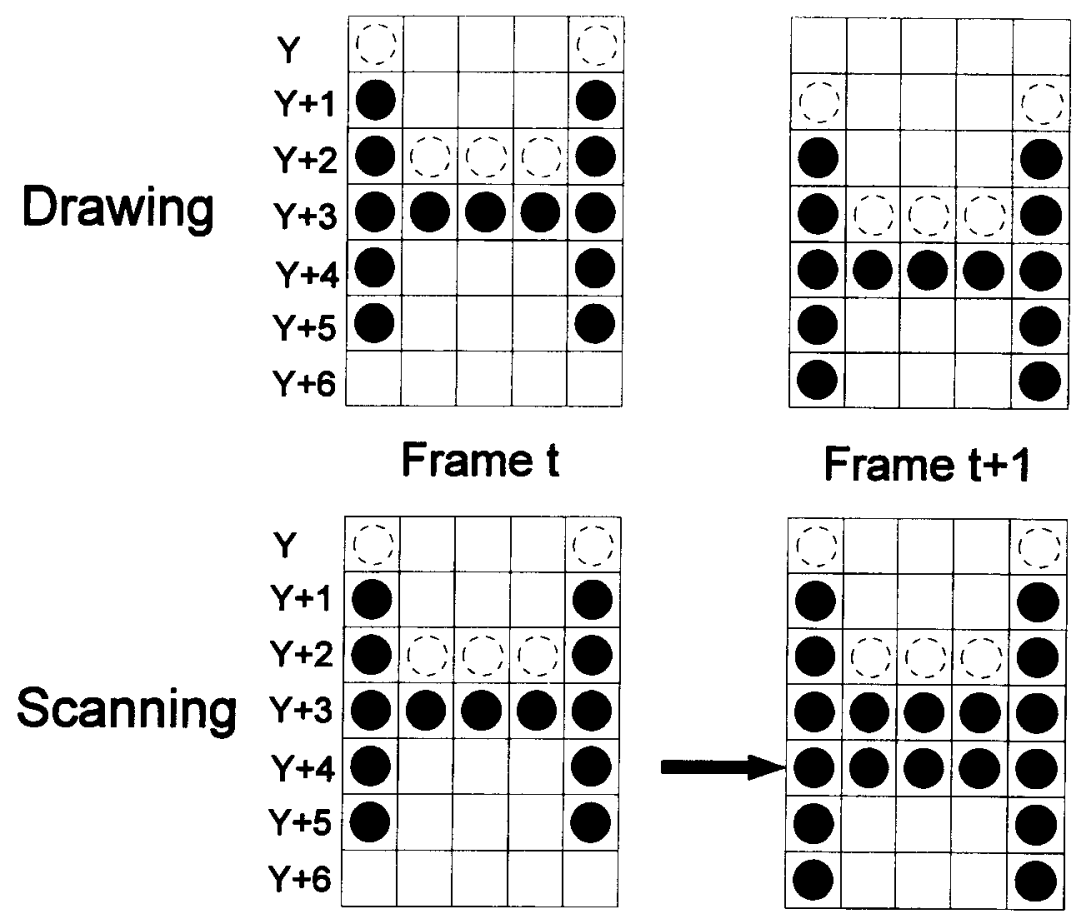

Figure 1. Example of how not linking to the retrace can result in distortions in motion displays. The upper panel shows how the stimulus is put into video memory. The lower panel shows how it appears on screen.

ducing apparent motion if the temporal and spatial conditions are right. To present smooth motion displays, both spatial and temporal resolutions need to be high, since jumps across too large a spatial or temporal gap make the movement look jerky or even abolish it (Wertheimer, 1912). The upper panel in Figure 1 shows how, on frame $t$, an $\mathrm{H}$ was drawn into video memory, starting on line $\mathrm{Y}+$ 1 and accompanied by an outline mask. On frame $t+1$, the same $\mathrm{H}$ is drawn, starting from $\mathrm{Y}+2$, and the mask effectively erases the remains of the previous $\mathrm{H}$. So in video memory, the $H$ has moved one pixel. The lower panel shows what can happen if drawing is not linked to the retrace. Frame $t$ correctly shows the $\mathrm{H}$ as we have put it in video memory. At frame $t+1$, however, the electron beam has started scanning at an arbitrary line $(Y+4$ in this case). The display first shows the bottom half of frame $t+1$, before the top half of frame $t$ has been erased. This means that the $\mathrm{H}$ will appear to change shape for a full scan period, which is usually clearly visible as a local flicker or as ghosting. Fortunately, there are routines to get the drawing in line with the scan rate (see, e.g., Haussmann, 1992, for an overview). These routines simply wait for the retrace and give a signal when the electron beam has returned to the upper left corner. This is the signal to start drawing the next frame.

The above describes how important it is that drawing commands are not issued until the electron beam flies back to the top. However, it is equally important that all the drawing commands have been completed by the time the electron beam starts to display the next frame. If not, again, either flicker or stimulus ghosting could result, because the electron beam would be drawing the stimuli to screen before their drawing operations had been fully issued. Thus, in practice, there is only one safe moment to issue the drawing commands, and that is during the retrace period. This means that the algorithm has to be very fast.

One way of ensuring that the consecutive frames in a motion sequence are generated on time is drawing the stimuli in advance, into a number of nondisplayed video pages, and then swapping the pages displayed by the monitor (again ensuring that page swapping is synchronized with the video retrace). Alternatively, one could use windowing techniques, in which the stimulus is essentially written to one memory page (again, in advance), which is then read, starting from different addresses. However, it can be the case that the number of video pages is insufficient for the number of stimulus frames that have to be presented sequentially. For instance, with displays of moving stimuli, a large number of frames, each containing small displacements of the stimuli, may be necessary in order to produce smooth continuous motion. Memory windowing further restricts the types of motion displays, in that the entire display can only rigidly move in a single direction (see Cox, 1997, for a review of the merits and demerits of the various motion techniques). The present paper is concerned with a very flexible technique, in which the drawing algorithm creates the stimuli on line in video memory before the electron beam has 


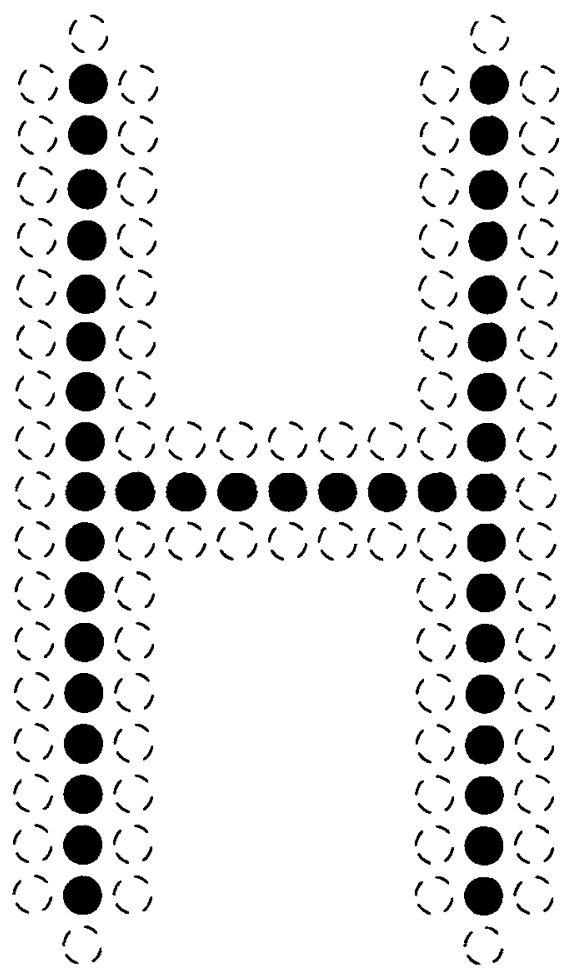

Figure 2. The pixel layout of an $H$ stimulus, as used by Watson and Humphreys (1997). The $H$ could randomly move in any of four directions and was, therefore, accompanied by a full mask (the dotted pixels), effectively erasing residues of previous frames.

started to trace the stimuli on the computer monitor. This way, any type of motion can be generated, provided that the drawing algorithm is fast enough to produce the next frame before the electron beam starts to trace it. In addition, only a single video page is required, which may often allow a higher spatial or color resolution to be used.

We present a simple method to speed up on-line drawing in certain types of displays, which has proven useful in increasing spatiotemporal resolution in our own motion experiments. The method involves the replacement of horizontal and vertical lines with thin rectangles. The improvements are especially apparent with horizontal lines.

\section{Speeding Up Line Drawing}

Recent experiments in our laboratory involved rather complex stimulus displays, based on those used by Watson and Humphreys (1997; see Figure 2). The experimental program was written in Borland Turbo Pascal 7.0 and made extensive use of the Genus Microcomputing GX Graphics libraries to generate the stimuli. On each trial, up to 16 letter Hs were randomly positioned across the screen, each of them randomly moving in one of four directions (north, east, south, and west), without colliding into other Hs. The flexible (i.e., random) assignment of position and direction to each item on each trial made on-line stimulus generation the most economical option. Further- more, a high spatial resolution and a spatiotemporal resolution of 1 pixel per scan period were required, to match with the previous experiments. Figure 2 shows how each $\mathrm{H}$ was drawn. Since motion could now be in any of four directions, a full mask was necessary around each $\mathrm{H}$, to erase the remains of old frames. The extra motion calculations, as well as the extra lines and rectangles of the mask, increased the total drawing time, so much so that a single frame could not be drawn in video memory before the electron beam started to display that frame on the screen, resulting in local flickering. ${ }^{1}$ In order to preserve the highest resolution, we needed to speed up the algorithm.

In our subsequent optimization attempts, we discovered that using the Rectangle and Bar commands to draw horizontal and vertical lines, instead of the Line command, resulted in the stimuli being drawn faster. Drawing a horizontal or a vertical line with a Rectangle command is very simple: One just replaces the line statement [e.g., $\operatorname{Line}(\mathrm{x} 1, \mathrm{y} 1, \mathrm{x} 2, \mathrm{y} 1)]$ with a rectangle statement of the same coordinates [e.g., Rectangle $(x 1, y 1, x 2, y 1)]$. We replaced all the lines with rectangles in our moving Hs displays, which gained the few extra milliseconds needed to draw the frame in video memory before any of the stimuli were traced on-screen by the electron beam.

Computer scientists have focused on improving the speed of algorithms that generate graphical primitives, such as lines and circles (e.g., Bresenham, 1965; Wu \& Rokne, 1987; Wyvill, 1990), and some programmers have suggested writing separate algorithms for special cases, such as horizontal and vertical lines (e.g., Ammeraal, 1992; see the General Discussion section). However, to our knowledge, direct comparisons between existing drawing commands designed for different graphics purposes (such as lines and rectangles) have never been reported in the relevant literature. Also, a search on the Internet and through computer graphics newsgroups yielded no hits. Yet, we will show that significant improvements in drawing speed can be made by creative use of exisiting drawing commands - that is, without having to resort to low-level programming techniques.

In the remainder of this paper, we report on our further exploration into how much can be gained by using a different set of commands for drawing lines. We designed a simple program that recorded the time needed to draw horizontal and vertical lines of various lengths in the Genus GX Graphics library and in the standard Borland Pascal 7.0 Graph library. The program was run in several different graphics modes and on six different machines. The crucial manipulation was between two methods of drawing, depending on which graphics library was used. In the case of the Genus library, we compared grDraw$\operatorname{Line}(\mathrm{x} 1, \mathrm{y} 1, \mathrm{x} 2, \mathrm{y} 2)$ to $\operatorname{grDrawRect}(\mathrm{x} 1, \mathrm{y} 1, \mathrm{x} 2, \mathrm{y} 2$, grOutline). Normally, these commands are used to draw a single line and a rectangular outline, respectively, but we used the rectangle command to draw lines (as was described above). In the case of the standard Borland library, we compared $\operatorname{Line}(\mathrm{x} 1, \mathrm{y} 1, \mathrm{x} 2, \mathrm{y} 2)$ to $\operatorname{Bar}(\mathrm{x} 1, \mathrm{y} 1, \mathrm{x} 2, \mathrm{y} 2)$. The Bar command effectively draws a filled rectangle 
Table 1

Hardware Configurations

\begin{tabular}{ll}
\multicolumn{1}{c}{ Processor } & \multicolumn{1}{c}{ Video Card } \\
\hline 1. Pentium 200 MMX & STB Nitro 3D, 4 MB \\
2. Pentium 60 & ATI Mach64, 2 MB \\
3. Pentium 120 & ATI Mach64, 4 MB \\
4. Pentium 120 & ATI Mach64, 2 MB \\
5. Pentium 166 MMX & Hercules Stingray 64, 1 MB \\
6. Pentium 233 MMX & Cirrus Logic, 2 MB \\
\hline
\end{tabular}

Note--All the machines were running under MS-DOS 6.22 , except numbers 5 and 6, which ran under Windows 95 (in DOS mode).

but can be used to draw horizontal and vertical lines equally well. ${ }^{2}$ To avoid confusion, we will use the term line to refer to the line image (i.e., as it actually appeared on the screen) and the terms Line and Rectangle/Bar to refer to the drawing method (thus, a line can be drawn by both Line and Rectangle/Bar).

\section{METHOD}

We used separate programs for each combination of graphics library and graphics mode, to minimize memory load and maximize transparency. We used the Genus GX Graphics and Borland Graph libraries, and graphics modes varied from low-resolution EGA and VGA $(640 \times 200 \times 16$ to $640 \times 480 \times 16)$ to high-resolution SVGA and VESA $(640 \times 480 \times 256$ to $1,024 \times 768 \times 256)$. Each program drew both horizontal and vertical lines of various lengths (5-200 pixels, step 5), starting from the upper left corner of the screen $(0,0)$. Each line was drawn 10,000 times, and drawing time was recorded at $1-\mathrm{msec}$ resolution. Drawing times were recorded in an array and later stored on disk. All the programs ran on various clean-booted MS-DOS 6.22 machines (i.e., with minimal start-up configurations) and on two Windows 95 machines in MS-DOS mode (see Table 1). These hardware configurations are actually used by experimenters in our lab.

\section{RESULTS}

\section{General Effects}

Graphics modes. There was no effect of resolution within similar graphics modes. For instance, in VESA mode with $1,024 \times 768$ resolution, lines were drawn as quickly as in VESA $640 \times 480$ mode. Also, we found no differences between low-resolution EGA and VGA modes (i.e., up to $640 \times 480 \times 16$ ). We therefore combined the data from similar modes within a library into (1) a lowresolution EGA/VGA group (Genus and Borland), (2) an SVGA group (Borland only), and (3) a VESA group (Genus and Borland). In both libraries, the VESA modes produced the fastest drawings, whereas the SVGA mode (Borland only) was overall the slowest.

Graphics library. In general, lines drawn using the Genus libraries were considerably (up to 10 times) faster than those drawn using the Borland library. However, relatively more time could be gained by using the Rectangle/ Bar method with the Borland library than with the Genus library (see below).
Line length. In all modes, longer lines took longer to draw. Drawing a 5-pixel line 10,000 times is usually done within $500 \mathrm{msec}$, whereas doing the same for 200 -pixel lines may take up to more than $5 \mathrm{sec}$ in the vertical Line case.

Hardware configuration. Not surprisingly, there were vast differences in drawing speed between the different hardware configurations (with faster CPUs and video cards generally yielding faster drawing times). Moreover, in some cases, hardware specification interacted with the effects above. Since the hardware technology is rapidly changing and the reader's configuration is probably different from ours, we will not present the hardware effects in detail. We recommend that the interested reader run programs similar to ours, in order to determine the absolute gains and any interactions on their particular hardware configuration. For the same reason, we do not further present absolute drawing times. Instead, we calculated the relative gains and losses that could be made by using the Rectangle/Bar command. In terms of this relative measure (see below), performance turned out to be quite consistent across the different hardware setups.

\section{Effects of Drawing Method: Horizontal Lines}

To gain more insight into the pattern of results, we created an index of the relative efficiency of the different drawing methods. We took the Line drawing times $\left(T_{1}\right)$ as the baseline level and calculated the relative gain (in percentages) for the Rectangle/Bar drawing times $\left(T_{\mathrm{rb}}\right)$, separately for each graphics mode (EGA/VGA, SVGA, VESA) and library (Genus, Borland). The gain is calculated as follows:

$$
\text { Gain }=\left(\left(T_{\mathrm{rb}}-T_{1}\right) / T_{1}\right) * 100(\%) .
$$

This measure was subsequently averaged across hardware configurations. Figures 3 and 4 show the efficiency of the Rectangle/Bar command, relative to the Line command, for different line lengths. The patterns of performance differed between the graphics modes, the two libraries, and occasionally the hardware. We will discuss these below.

Borland. In Figures $3 a-3 c$, the relative performance of Bar is plotted for the different Borland graphics modes. The results show that, within the Borland Pascal Graph library, a greater than $90 \%$ increase in drawing speed can be achieved if a horizontal Bar is used instead of a horizontal Line, in both EGA/VGA and SVGA modes (e.g., where 10,000 Lines took 2,500 msec, 10,000 Bars took only $250 \mathrm{msec}$; Figures $3 \mathrm{a}$ and $3 \mathrm{~b}$ ). In the SVGA mode, the gain is already more than $50 \%$ at very short lines (5 pixels). In the VESA mode (Figure 3c), the pattern of performance is somewhat different. There seem to be two groups of hardware configurations, each with its own pattern of savings and losses. In Group I (machines 1, 5, and 6; see Table 1), there is a headstart for short lines (up to 25 pixels), but then the advantage turns into a loss, which stays fairly constant with increasing line length. 

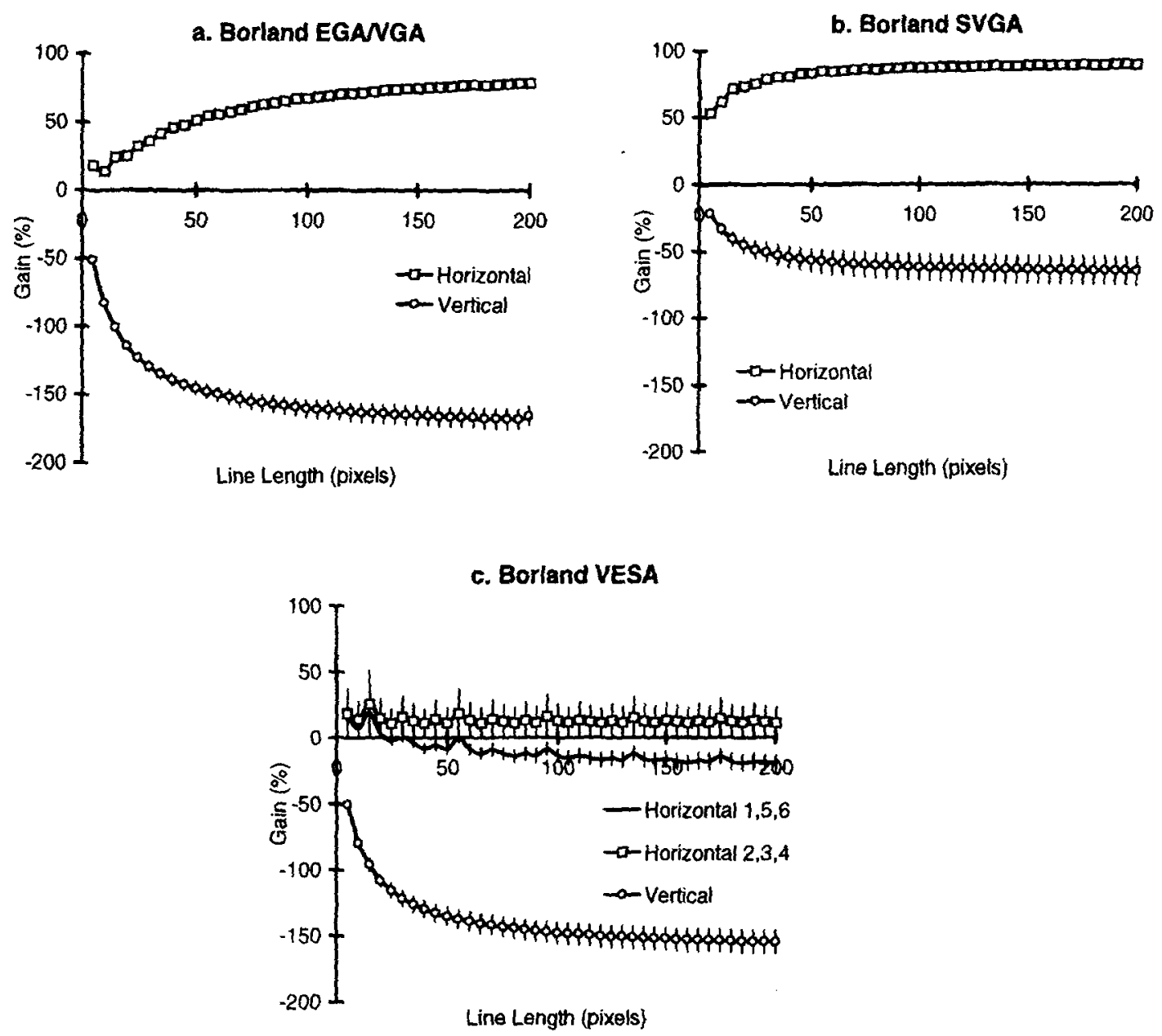

Figure 3. Data for the Borland Graph unit. The $y$-axis represents the relative gain in efficiency (i.e., drawing time) of the Bar method, relative to the Line method. Error bars represent the standard error variation across hardware. The numbers in panel $3 \mathrm{c}$ correspond to the hardware configurations listed in Table 1 . Note that error bars in panels $3 \mathrm{a}$ and $3 \mathrm{~b}$ are virtually absent for horizontal lines, indicating the high consistency of the findings across hardware.

Group II (machines 2, 3, and 4), however, stabilizes above zero, resulting in a small but constant advantage for the Bar command (around 20\%).

Genus. In Figures $4 \mathrm{a}$ and $4 \mathrm{~b}$, performance of Rectangles, relative to Lines, is plotted. In the EGA/VGA mode (Figure 4a), there is nothing to be gained (or lost) by using Rectangles instead of Lines. In the VESA mode, however, savings are considerable, starting at $40 \%$ for short lines and slowly dropping to $30 \%$ for longer lines. AIthough, relative to the Borland library, there is more variation across hardware, the pattern of performance proves to be consistent.

\section{Drawing Method: Vertical Lines}

Borland. Replacing vertical Lines with Bars produced effects opposite to those for horizontal lines in all graphics modes (Figures 3a-3c). Bars substantially decreased the algorithm's speed, often by more than $150 \%$ (e.g., if
10,000 Lines took $1,000 \mathrm{msec}, 10,000$ Bars would take $2,500 \mathrm{msec}$ ). The effects were consistent across hardware, with minimal variation.

Genus. In the EGA/VGA mode (Figure 4a), performance was very similar to that for the Borland library, in that vertical Rectangles are generally much slower than Lines (up to about $100 \%$ ), with some variation across hardware. In the VESA mode, however, there were two main groups of hardware (Figure 3b). Group I (machines 1, 3, and 6; see Table 1) shows a headstart for Rectangles up to a length of about 125 pixels, after which savings become virtually negligible or even turn into a loss. Group II (machines 2, 4, and 5) demonstrates a substantial loss for longer lines, but relative savings for short lines (up to 25 pixels). Note that there is some overlap between these groups of hardware and those we distinguished for horizontal Bars in the Borland VESA mode, but they are not identical. 

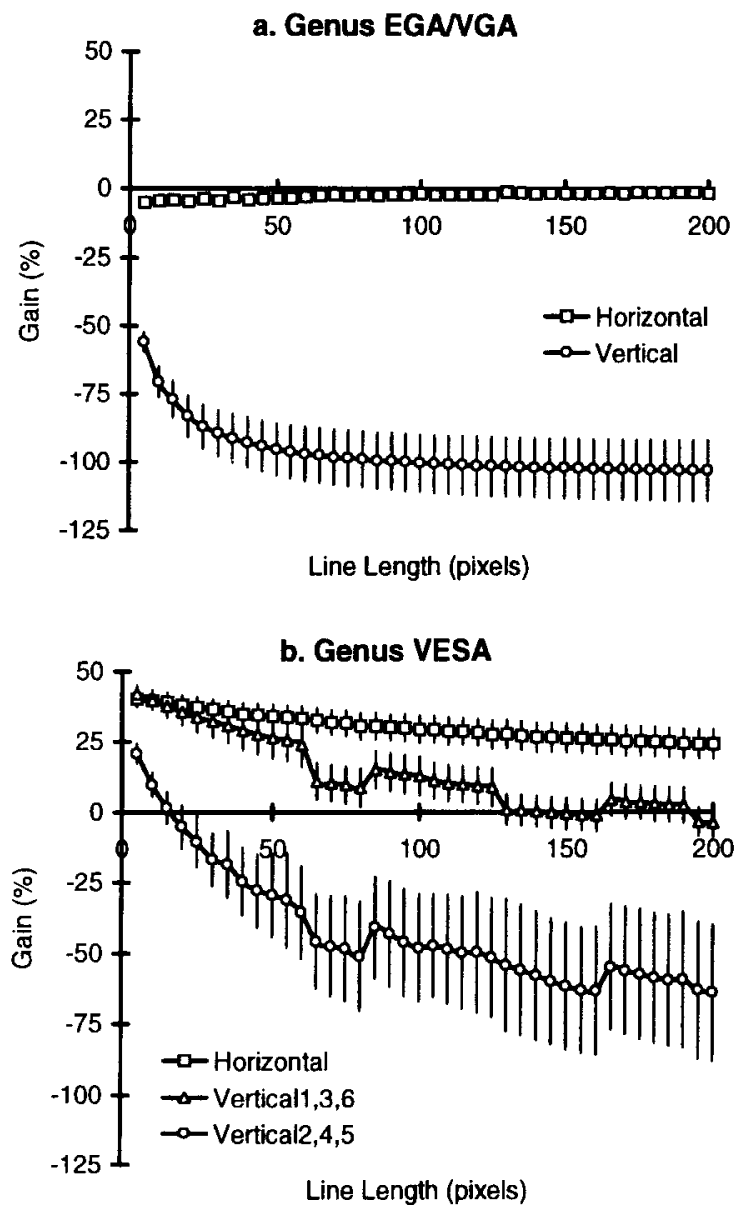

Figure 4. Data for the Genus Graph unit. The $y$-axis represents the relative gain in efficiency (i.e., drawing time) of the Rectangle method, relative to the Line method. Standard error bars represent the variation across hardware. The numbers in panel $\mathbf{4 b}$ correspond to the hardware configurations listed in Table 1. Error bars in panel $4 \mathrm{a}$ are virtually absent for horizontal lines, because of little variation across hardware.

\section{DISCUSSION}

The main finding of this study is that considerable time savings (up to $90 \%$ ) can be made by using a Rectangle/Bar command instead of a Line command to draw stimuli. This is mainly the case for horizontal lines (although not in the Genus EGA/VGA modes). Replacing vertical Lines with Bars/Rectangles generally results in time losses, although on some occasions, savings can be made (in the Genus VESA modes). The advantage for horizontal lines is highly consistent across different hardware setups, except for some variation in the VESA modes. We measured absolute savings of more than $3 \mathrm{sec}$ for 10,000 line drawings in the Borland SVGA mode, amounting to $0.3 \mathrm{msec}$ per line (averaged across hardware). That this is not negligible has already been proven in our motion experiments, in which stimulus frames consisted of more than 200 line segments each ( $16 \mathrm{Hs}$ and their masks). In this experiment, we used the much faster Genus library, which produces relatively smaller savings (in the order of $10 \mathrm{~s}$ of $\mu \mathrm{s} /$ line). Still, replacing all Line with Rectangle commands resulted in the speed increase that was required to remove ghosting and flicker from the displays, while allowing the maximum spatiotemporal resolution to be preserved.

The results also show that savings often depend on hardware configuration, graphics mode, and their interactions with line length. We recommend, therefore, that before applying the Rectangle/Bar substitution method, the reader check the pattern of performance of his or her particular setup. Furthermore, the findings may not be confined to a particular programming language, such as Turbo Pascal. We found similar findings with programs compiled from $\mathrm{C}++$.

There are several explanations for the fact that Rectangles and Bars are often faster, but none of these explanations can account for all the data. First, many graphics packages rely on Bresenham's (1965) basic algorithm for plotting straight lines. This algorithm has generally proven to be the fastest, as it can calculate pixel positions representing straight lines of any slope, without resorting to (relatively slow) multiplication and divisions (see Wyvill, 1990, for a summary on recent improvements of Bresenham's algorithm). Although fast, the fact that it must be able to deal with lines of any slope (i.e., in which both $x$ and $y$ positions need to be updated, often at different rates) does not make it the most efficient algorithm for horizontal or vertical lines (in which either $x$ only or $y$ only needs updating). Some authors therefore suggest writing separate algorithms for horizontal and vertical lines (e.g., Ammeraal, 1992). A Rectangle or Bar command can be more efficient, because there are no slopes involved. When used to draw a straight line, only one direction needs to be updated. The fact that the savings are much more apparent for horizontal lines than for vertical lines could be explained by assuming that the Rectangle/ Bar algorithms choose the horizontal $(x)$ direction first. However, such an account does not explain why, on some machines, the benefits for horizontal lines suddenly turn into losses and why, in some configurations, but not in others, there are savings to be made even with vertical lines. We suggest that the reason for such variation is likely to be found in the particular electronic architecture of video cards and the way in which they operate under certain video modes. Whereas the EGA, VGA, and SVGA modes operate according to rather strict hardware standards (without graphic acceleration), the VESA standard only dictates which types of input and output the display adapter should comply to. The way in which the hardware operates in VESA mode differs from manufacturer to manufacturer and from model to model (Rosch, 1997, p. 753). For instance, most modern VESA adapters make extensive use of various hardware acceleration techniques to speed up the drawing of graphic primitives, such as lines and boxes (since these are important primitives in the Windows environment). Our data seem to support 
the conclusion that the qualitative differences in performance are mainly caused by hardware variations, since they are affected by different machine configurations, but primarily so in the VESA mode (Figures $3 \mathrm{c}$ and $4 \mathrm{~b}$ ).

Whatever the cause, the effect is real and can result in substantial time savings, even on fast Pentium-processorbased machines. We hope that the method presented here can be of some assistance in optimizing drawing algorithms for the display of visual stimuli.

\section{REFERENCES}

AmmeraAl, L. (1992). Programming principles in computer graphics (2nd ed.). New York: Wiley.

BaCh, M., Meigen, T., \& Strasburger, H. (1997). Raster-scan cathoderay tubes for vision research: Limits of resolution in space, time and intensity, and some solutions. Spatial Vision, 10, 403-414.

Bitterman, M. E., Krauskopf, J., \& HochberG, J. E. (1954). Threshold for visual form: A diffusion model. American Journal of Psychology, 67, 205-219.

Bresenham, J. E. (1965). Algorithm for computer control of a digital plotter. IBM Systems Journal, 4, 25-30.

Cox, M. J. (1997). Making things move: The options for computerbased displays. Spatial Vision, 11, 3-17.

HaussmanNN, R. E. (1992). Tachistoscopic presentation and millisecond timing on the IBM PC/XT/AT and PS/2: A Turbo Pascal unit to provide general-purpose routines for CGA, Hercules, EGA, and
VGA monitors. Behavior Research Methods, Instruments, \& Computers, 24, 303-310.

Rosch, W. L. (1997). Hardware Bible. Indianapolis: Sams.

Tanner, P. P., Jolicoeur, P., Cowan, W. B., Booth, K., \& Fishman, F. D. (1989). Antialiasing: A technique for smoothing jagged lines on a computer graphics image - an implementation on the Amiga. Behavior Research Methods, Instruments, \& Computers, 21, 59-66.

Watson, D. G., \& HumphreYs, G. W. (1997). Visual marking: Prioritising selection for new objects by top-down attentional inhibition of old objects. Psychological Review, 104, 90-122.

Wertheimer, M. (1912). Experimentelle Studien über das Sehen von Bewegung [Experimental studies on the perception of motion]. Zeitschrift für Psychologie, 61, 161-265.

Wu, X. L., \& RoKNE, J. G. (1987). Double-step incremental generation of lines and circles. Computer Vision Graphics \& Image Processing, 37, 331-344.

WyvilL, B. (1990). Symmetric double step line algorithm. In A. S. Glassner (Ed.), Graphics GEMS (pp. 101-104). Boston: Academic Press.

\section{NOTES}

1. Alternatively, a separate mask could have been drawn for each direction, but the extra code necessary produced even greater time costs.

2. The Borland Graph unit also contains a Rectangle(x1, y1, x2, y2) procedure, but we found out that Rectangle is always slower than Line.

(Manuscript received June 1, 1998; revision accepted for publication August 19, 1998.) 